Phys. Rev. E 65 (2002)

\title{
Electric microfield distributions in electron-ion plasmas
}

\author{
Alexander Y. Potekhin ${ }^{*}$ \\ Ioffe Physical-Technical Institute, 194021 St. Petersburg, Russia \\ Gilles Chabrien \\ Ecole Normale Supérieure de Lyon, CRAL (UMR CNRS No. 5574), 69364 Lyon Cedex 07, France \\ Dominique Gillesf \\ Commissariat à l'Energie Atomique, BP 12, 91680 Bruyères-le-Châtel, France
}

(Dated: 5 July 2001, accepted 17 December 2001)

\begin{abstract}
The low-frequency electric microfield distribution in a Coulomb plasma is calculated for various plasma parameters, from weak to strong Coulomb coupling and from zero to strong electron screening. Two methods of numerical calculations are employed: the adjustable-parameter exponential approximation and the Monte Carlo simulation. The results are represented by analytic fitting formulas suitable for applications.

PACS numbers: 52.27.Gr, 52.65.Pp
\end{abstract}

\section{INTRODUCTION}

Because of the Stark effect, stochastic electric microfields influence optical and thermodynamic properties of a plasma. First, they affect the profiles of spectral lines and effectively lower photoionization thresholds of atoms and ions immersed in a plasma [1, 2]. A comparison of experimental and theoretical widths and shapes of the Stark-broadened spectral lines is widely used for plasma diagnostics (e.g., Refs. [3, 4]). Second, in some theoretical models of the plasma equation of state (e.g., Refs. [5, 6]), the microfield distribution is used in order to calculate occupation numbers of the bound species (although such a calculation is not free from principal difficulties, as discussed in Ref. [7]). It was shown recently [6] that a more accurate description of the microfields entails a considerable improvement of the equation-of-state model.

In many cases, the microfield perturbation can be treated as quasistationary. Then the problem is reduced to determination of the probability distribution of the low-frequency component of perturbing electric fields (e.g., Ref. [8]), associated with a stochastic distribution of perturbing ions, whereas the electrons can be assumed to adjust instantaneously to a configuration of the ions. The low-frequency microfields are appropriate to use in the equation of state models [6] and in calculation of spectroscopic line profiles for those radiative transitions whose frequency does not exceed the typical frequency of microfields produced by thermal fluctuations of the electron density. For example, Stehlé and Jacquemot [9] used the model microfield method to analyze the line shapes and line dissolution in hydrogen plasma spectra.

\footnotetext{
*Electronic address: palex@astro.ioffe.rssi.ru

${ }^{\dagger}$ Electronic address: chabrier@ens-lvon.fr

‡Electronic address: Dominique.Gilles@cea.fr
}

Holtsmark [10] has derived the microfield distribution function assuming that the ions are not correlated and the electron screening is negligible. This assumption is justified for very hot or rarefied plasmas, for which the Coulomb coupling parameter

$$
\Gamma=\frac{(Z e)^{2}}{a k_{B} T} \approx \frac{1.25 \times 10^{4} \mathrm{~K}}{T} n_{20}^{1 / 3} Z^{5 / 3}
$$

is close to zero. Here, $Z e$ is the ion charge, $T$ is the temperature, $k_{B}$ is the Boltzmann constant, $a=\left(\frac{4 \pi}{3} n_{i}\right)^{-1 / 3}$ is the ion sphere radius, $n_{i}$ is the ion number density, and $n_{20}$ is the electron number density $\left(n_{e}=Z n_{i}\right)$ in units of $10^{20} \mathrm{~cm}^{-3}$. As we demonstrate below, the Holtsmark approximation is inaccurate already at $\Gamma \sim 0.1$. In modern plasma experiments, $\Gamma$ may approach unity, whereas in stellar matter it can be much larger. In these cases, correlations of plasma particles should not be neglected.

Various approximations were developed in the past in order to take the ion correlations into account. If $\Gamma \lesssim 1$, one may use the methods of Baranger and Mozer 11] or Hooper 112, 13 based on a cluster expansion in powers of density. The electron screening is usually described by a Debye-like (Yukawa) effective potential, introduced in the context of microfield distributions by Hoffman and Theimer [14. In the limit of extremely strong coupling, $\Gamma \gg 10$, and without screening, the harmonic oscillator model by Mayer [15] is applicable, in which every ion is assumed to oscillate independently of the others around its equilibrium position at the ion-sphere center.

The first theory capable to provide reliable numerical results for strongly coupled plasmas with electron screening proved to be the adjustable-parameter exponential approximation (APEX), based on a special parametrization of the electric microfield $\boldsymbol{E}$ produced on a selected test particle (neutral or charged "radiator" of charge $Z_{r}$ ) which undergoes the influence of charged plasma particles ("perturbers" of species $\sigma$ and of charge $Z_{\sigma}$ ). This method has been developed for Coulomb systems [16] and adapted for screened Coulomb systems and ion mixtures 
[8, 17. It involves non-interacting quasiparticle representation of the electron-screened ions, designed to yield the correct second moment of the microfield distribution [18:

$$
\langle\boldsymbol{E} \cdot \boldsymbol{E}\rangle=\frac{4 \pi n_{i} k_{B} T}{Z_{r}} k_{s}^{2} \sum_{\sigma} c_{\sigma} Z_{\sigma} \int_{0}^{\infty} d r r e^{-k_{s} r} g_{\sigma}(r),
$$

where $g_{\sigma}(r)$ and $c_{\sigma}$ denote the radial distribution function (RDF) and the relative abundance of species $\sigma$, respectively, and where $k_{s}$ is an effective electron screening wave-number. After introducing the effective singleparticle field in the form

$$
\epsilon_{\sigma}^{*}=Z_{\sigma} e \frac{\left(1+\alpha_{\sigma} r\right)}{r^{2}} e^{-\alpha_{\sigma} r}
$$

the adjustable parameters $\left\{\alpha_{\sigma}\right\}$ are chosen to satisfy the condition

$$
4 \pi \int \epsilon^{2} P_{\mathrm{APEX}}(\epsilon) d \epsilon=\langle\boldsymbol{E} \cdot \boldsymbol{E}\rangle
$$

The expression on the left-hand side of this equation contains the parameters $\left\{\alpha_{\sigma}\right\}$ to be determined, whereas the right-hand side can be evaluated using Eq. (2), if the RDF is known. The RDF thus provides a scheme for evaluating the APEX microfield distribution and is a central ingredient whose accuracy determines the one of the APEX microfield results. In our implementation of the APEX technique, we have used the hypernettedchain RDF calculations 18, 19, 20].

On the other hand, with the advent of powerful computers it is now possible to calculate the microfield distribution from Monte Carlo (MC) or molecular-dynamics simulations of plasmas with the minimum of simplifying assumptions (e.g., Refs. [21, 22, 23, 24, 25]). Moreover, the latter methods allow one to study the effects of microfield nonuniformity [26, 27] and to simulate highfrequency microfield distributions in electron-ion plasmas (e.g., Ref. [28]). The MC technique is based on a numerical simulation of space configurations of a system of particles, whereas the molecular-dynamics technique traces the time evolution of the system. For the low-frequency microfield, dynamical effects are unimportant, and the two methods yield the same results, as demonstrated, e.g., in Ref. [27]. Therefore it is sufficient to use the MC method in this case.

With these powerful tools, the microfield distribution can be calculated now for any practically important combination of plasma parameters. However, plasma spectroscopy and equation-of-state models require knowledge of this distribution at many different points or even in continuous areas of the plasma parameter space. In this case, either extensive numerical tables or approximate analytic expressions are necessary.

We present results of calculations of the low-frequency microfield distribution function at a neutral and charged plasma point for various values of $\Gamma$ ranging from 0 to
100 and for various values of an effective electron screening length. We consider plasmas composed of a single species of ions; in particular, in the case of a charged test particle, its charge is assumed to be equal to that of perturbers. The calculations are performed mainly by the MC method; for comparison we have done also APEX calculations. We also present analytic formulas which reproduce the calculated electric microfield probability distributions with an accuracy comparable to small differences between the MC and APEX results.

In the next section, we describe basic assumptions used in our calculations and write down some asymptotic results. In Sec. III, we present results of numerical calculations and analytic approximations for microfield distributions produced at a neutral or charged point by ions interacting via unscreened or screened Coulomb potentials. The results are summarized in Sec. IV.

\section{METHOD}

\section{A. Basic assumptions}

We consider a nonrelativistic, isotropic, overall neutral plasma at the thermodynamic equilibrium. The ions are assumed to be classical and pointlike. The electric field created at a point $\boldsymbol{r}$ by an ion placed at $\boldsymbol{r}_{i}$ equals $\tilde{\boldsymbol{E}}\left(\boldsymbol{r}_{i}-\right.$ $\boldsymbol{r})=-(Z e)^{-1} \nabla V\left(\left|\boldsymbol{r}_{i}-\boldsymbol{r}\right|\right)$, where $V(r)$ is an effective pair potential. This potential is taken in the Debye-Hückel (or Yukawa) form:

$$
V(r)=(Z e)^{2} \frac{e^{-k_{s} r}}{r} .
$$

In the linear approximation, a test charge $q$ embedded (at $\boldsymbol{r}=0$ ) creates perturbation of electron number density $\tilde{n}_{e}(r)=\left(\partial n_{e} / \partial \mu\right) \tilde{\mu}(r)$, where $\tilde{\mu}(r)=-e \phi(r)$ is the perturbation of the electron chemical potential $\mu$, and $\phi(r)$ is the excess electrostatic potential determined by the Poisson equation $\nabla^{2} \phi(r)=-4 \pi\left[q \delta^{3}(\boldsymbol{r})-e \tilde{n}(r)\right]$. Thus in the linear (first-order perturbation) approximation $\left(\nabla^{2}+4 \pi e^{2} \partial n_{e} / \partial \mu\right) \phi(r)=-4 \pi q \delta^{3}(\boldsymbol{r})$, which leads to the well-known (e.g., Ref. [20]) expression for the effective screening wave number $k_{s}$ :

$$
k_{s}^{2}=4 \pi e^{2} \frac{\partial n_{e}}{\partial \mu}=\frac{e^{2}}{\pi \hbar^{3}}\left(2 m_{e}\right)^{3 / 2}\left(k_{B} T\right)^{1 / 2} I_{-1 / 2}(\chi),
$$

where $I_{\nu}(\chi)=\int_{0}^{\infty} x^{\nu} d x /\left(e^{x-\chi}+1\right)$ is the Fermi integral, and $\chi \equiv \mu / k_{B} T$ is determined from the equation

$$
I_{1 / 2}(\chi)=\pi^{2} \hbar^{3}\left(m_{e} k_{B} T\right)^{-3 / 2} n_{e} / \sqrt{2} .
$$

The solution of Eq. (7) and the right-hand side of Eq. (6) are given by accurate Padé approximations in Ref. [29].

In the limits of weak or strong electron degeneracy, $k_{s}$ tends to the inverse Debye length for the electrons or to the Thomas-Fermi wave number, respectively.

We adopt the conventional assumption that the potentials $V\left(\left|\boldsymbol{r}_{i}-\boldsymbol{r}\right|\right)$ are additive, which is strictly valid in the 
limit $k_{s} \rightarrow 0$. Then the electric field $\boldsymbol{E}(\boldsymbol{r})$ is also the sum of elementary electric fields $\tilde{\boldsymbol{E}}\left(\boldsymbol{r}_{i}-\boldsymbol{r}\right)$.

It is convenient to introduce the dimensionless field $\boldsymbol{\beta}$ and the screening parameter $s$ :

$$
\boldsymbol{\beta}=\left(a^{2} / Z e\right) \boldsymbol{E}, \quad s=a k_{s} .
$$

Also, $\tilde{\boldsymbol{\beta}}=\left(a^{2} / Z e\right) \tilde{\boldsymbol{E}}$.

In the canonical thermodynamic ensemble of $(N+1)$ particles, the probability density of the modulus of the field, $\beta=\left|\boldsymbol{\beta}\left(\boldsymbol{r}_{0}\right)\right|$, can be written as

$$
\begin{aligned}
P(\beta)= & \frac{4 \pi \beta^{2}}{\mathcal{Z}_{N+1}} \int \ldots \int \delta\left[\boldsymbol{\beta}\left(\boldsymbol{r}_{0}\right)-\sum_{i=1}^{N} \tilde{\boldsymbol{\beta}}\left(\boldsymbol{r}_{i}-\boldsymbol{r}_{0}\right)\right] \\
& \times e^{-W\left(\boldsymbol{r}_{0}, \boldsymbol{r}_{1}, \ldots, \boldsymbol{r}_{N}\right) / k_{B} T} d \boldsymbol{r}_{1} \ldots d \boldsymbol{r}_{N} .
\end{aligned}
$$

Here, $\mathcal{Z}_{N+1}=\int \ldots \int e^{-W\left(\boldsymbol{r}_{0}, \boldsymbol{r}_{1}, \ldots, \boldsymbol{r}_{N}\right) / k_{B} T} d \boldsymbol{r}_{1} \ldots d \boldsymbol{r}_{N}$ is the canonical partition function and $W$ is the potential energy of the configuration: $W\left(\boldsymbol{r}_{0}, \boldsymbol{r}_{1}, \ldots, \boldsymbol{r}_{N}\right)=$ $\frac{1}{2} \sum_{i \neq j} V\left(\left|\boldsymbol{r}_{i}-\boldsymbol{r}_{j}\right|\right)+V_{B}$, where $V_{B}$ is the potential energy of the background of electrons. Our goal is to calculate the function $P(\beta)$ in the thermodynamic limit $N \rightarrow \infty$. In the next paragraph we shall discuss how to perform this calculation.

\section{B. Monte Carlo technique}

In the numerical MC calculations, the coordinates of $(N+1)$ particles (one test particle and $N$ perturbers) are chosen in a cubic box of side length $L$ such that $(N+1) / L^{3}=n_{i}$. In order to include the effect of distant particles, the box is replicated by its "images" filling the space with the step $L$. The sum of the interaction potentials with all ion images is calculated using the Ewald technique [30] and, in order to ensure isotropy, the averaging over the complete solid angle is applied. As shown in Ref. [21, a result of this procedure is equivalent to the replacement of the potential (5) in the cube by an effective potential $V_{L}$,

$\frac{V_{L}(r)}{(Z e)^{2}}=\frac{e^{-k_{s} r}}{r}-\frac{C_{M}^{\prime}}{L} \frac{\sinh \left(k_{s} r\right)}{k_{s} r}+\frac{4 \pi}{k_{s}^{2} L^{3}}\left[\frac{\sinh \left(k_{s} r\right)}{k_{s} r}-1\right]$,

where $C_{M}^{\prime}$ is a numerical constant which tends to the Madelung constant (e.g., Ref. [31]) in the limit $k_{s} \rightarrow 0$. The total potential energy $W$ is obtained by the summation of $V_{L}$ over all pairs of particles.

During the MC run, an ion within the box and its displacement are chosen randomly. If the displacement brings the ion outside the box, the ion is replaced by its image. In strongly correlated plasmas, the displacement is limited by a maximum distance smaller than $L$, in order to avoid calculation of the energy for highly improbable configurations. First $10^{4}$ ion configurations are discarded in order to erase traces of the starting configuration. The state of equilibrium is searched by the
Metropolis algorithm: the energy difference $\Delta W$ is calculated between the consecutive configurations, and the new configuration is accepted definitely if this difference is negative and accepted with probability $e^{-\Delta W / k_{B} T}$ if $\Delta W$ is positive. The latter condition allows the system to escape from trapping in a local energy minimum. When the system approaches equilibrium, $W$ ceases to change appreciably. Then all equilibrium quantities depending only on particle positions (electric microfield, pair correlation function, etc.) can be calculated. In all our simulations, we try to get the maximum precision by minimizing the statistical errors. So we considered large boxes of particles (between $N=600$ and $N=800$ ) and, for each state $(\Gamma, s),(1-6) \times 10^{7}$ configurations were generated after equilibrium. This number of samples is large enough for the precision needed for all microfield distribution results discussed in this paper.

\section{Asymptotic and approximate expressions}

The described MC sampling procedure does not directly provide the probability density for extremely weak or strong fields, which are given by rare configurations. It is therefore useful to know asymptotic behavior of $P(\beta)$ in the limits $\beta \rightarrow 0$ and $\beta \rightarrow \infty$.

In the first case, the methods of Baranger and Mozer [11 and APEX 8, 16] show a parabolic dependence of $P(\beta)$ near the origin. This behavior is also visible on $\mathrm{MC}$ results. Thus we assume that $P(\beta) / \beta^{2}$ is constant near the origin.

In the case of very strong fields, exact analytic results are available for the unscreened Coulomb potential only. The Holtsmark distribution, valid at $\Gamma \rightarrow 0$ for any $\beta$, reads 10 .

$$
P_{H}(\beta)=\frac{2 \beta}{\pi} \int_{0}^{\infty} x \exp \left(-x^{3 / 2}\right) \sin (\beta x) d x .
$$

At $\beta \rightarrow \infty$, this distribution has the asymptote $P(\beta) \sim$ $1.496 \beta^{-5 / 2}$, which is close to the asymptote of the nearest-neighbor $(\mathrm{NN})$ field distribution [32]

$$
P_{\mathrm{NN}}(\beta)=1.5 \beta^{-5 / 2} \exp \left(-\beta^{-3 / 2}\right) .
$$

In the opposite limit of extremely strong correlations $(\Gamma \rightarrow \infty)$, the Mayer model [15] yields (for the charged test particle)

$$
P_{M}(\beta)=\sqrt{2 / \pi} \Gamma^{3 / 2} \beta^{2} \exp \left(-\Gamma \beta^{2} / 2\right) .
$$

Figure 1 illustrates the differences between various asymptotic theories (NN, Mayer model) and numerical results at finite $\Gamma$. Compared to the Holtsmark distribution (11), the most probable field values are shifted considerably to lower $\beta$, the shift being much larger at a charged point. The NN approximation (12) correctly describes the case of large $\beta$ for the neutral point but fails for the charged point or at small $\beta$. The Mayer distribution (13) fails to describe the high-field tail of $P(\beta)$ 


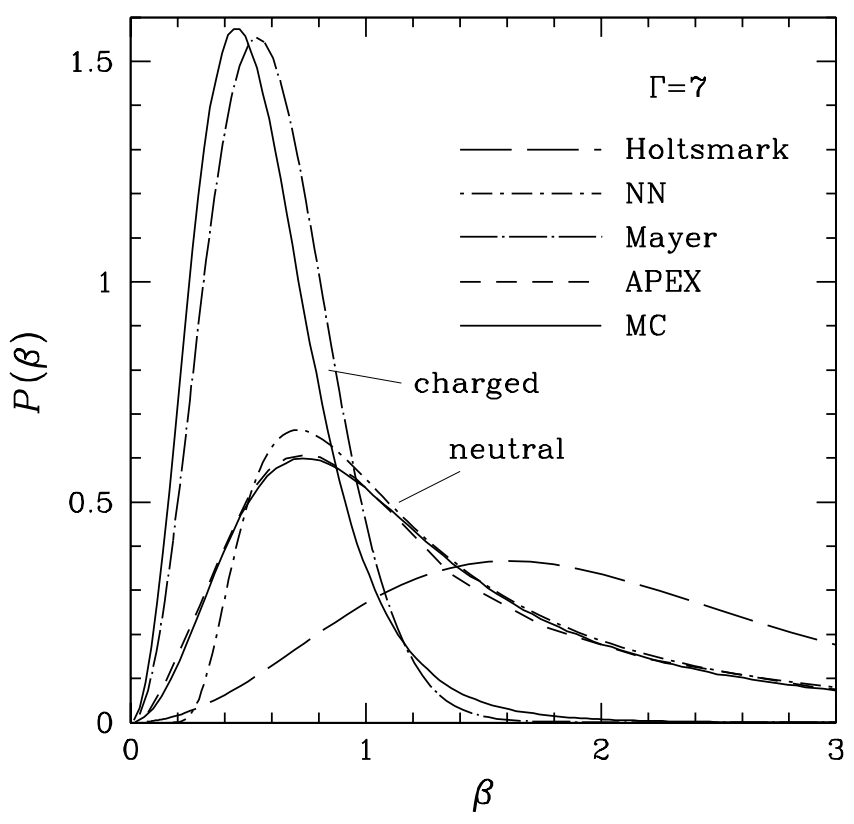

FIG. 1: Microfield distributions produced by ions interacting via the unscreened Coulomb potential in a plasma at $\Gamma=7$ at a neutral and charged point in the plasma. MC (solid lines) and APEX (short-dashed curve) numerical results are compared with analytic approximations by Holtsmark (long dashes), Mayer and nearest neighbor (dot-dashed lines).

but provides the most probable field which, at this $\Gamma$, is offset by tens percent only. At contrast, the APEX and $\mathrm{MC}$ results are in close agreement for $\beta<2$.

The Mayer model fails in the strong-field limit, because in this case one should consider a test ion which lies at a very short distance $r \propto \beta^{-1 / 2}$ from the nearest perturbing ion. Then the geometrical and Boltzmann factors give $P(\beta) \sim \beta^{-5 / 2} e^{-\Gamma \beta^{1 / 2}}$ at $\beta \rightarrow \infty$. In Appendix A we present derivation of a more accurate asymptotic expression, Eq. (A4), which was previously given in Refs. [24, 33] for the case of the Coulomb potential without screening. A generalization of Eq. (A4) provides an accurate functional form of the asymptotic behavior of $P(\beta)$ at large $\beta$ [24, 34]:

$$
P(\beta) \sim \tilde{K} \beta^{-5 / 2} \exp \left(-\tilde{\Gamma} \beta^{1 / 2}-\beta^{-3 / 2}\right)
$$

where $\tilde{K}$ and $\tilde{\Gamma}$ are adjustable parameters. In practice, $\tilde{\Gamma}$ is a free parameter, whereas $\tilde{K}$ can be determined from the normalization constraint. An example is given in Fig. 2. In this example, the value of $\tilde{\Gamma}$ is close to the exact $\Gamma$, but this is not the general case. However, as shown in Refs. [24, 34], for any simulation of practical interest, it is always possible to find appropriate $\tilde{\Gamma}$ and $\tilde{K}$ to fit the high-field tail of the microfield distribution.

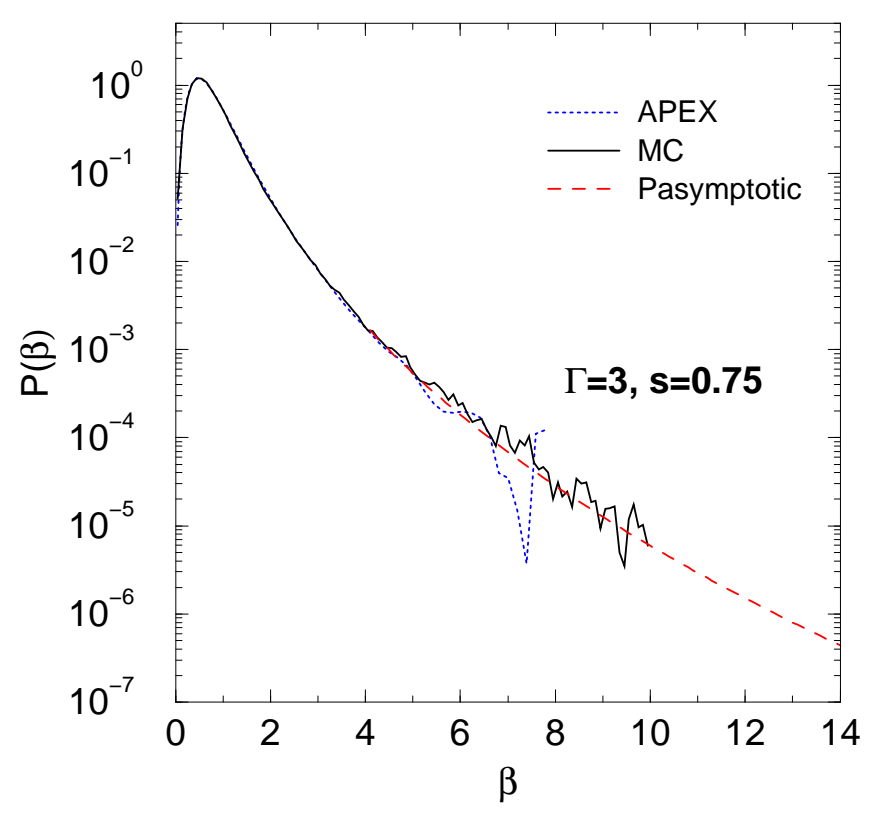

FIG. 2: Comparison, for $\Gamma=3$ and $s=0.75$, of probability density distributions $P(\beta)$ at a charged point, calculated by different methods: Monte Carlo (solid line), APEX (dotted line), and the asymptotic expression (14) (dashed line). Coefficients of the asymptotic expression have been fitted on MC points above the cut-off value $\beta=5: \tilde{\Gamma}=3.06$ and $\tilde{K}=31.011$. The asymptotic expression is a good representation of the high-field tail of $P(\beta)$ since it avoids the oscillations shown by the two other methods (MC: numerical statistical noise, APEX: Fourier transform oscillations).

\section{RESULTS}

\section{A. Coulomb potential}

In this section, we present results of $\mathrm{MC}$ and APEX calculations and analytic approximations for the microfield distribution brought about by the onecomponent plasma ions interacting via the Coulomb potential without screening. In this case, the probability density $P$ depends on $\beta$ and $\Gamma$.

\section{Neutral point}

First we consider the distribution of electric microfields applied to a neutral test particle embedded in a plasma. If the plasma is weakly coupled, $P(\beta)$ is given by the Holtsmark formula (11). In theoretical models (e.g., 河, [6]) one is often interested in the cumulative probability distribution defined as

$$
Q(\beta)=\int_{0}^{\beta} P\left(\beta^{\prime}\right) d \beta^{\prime} .
$$




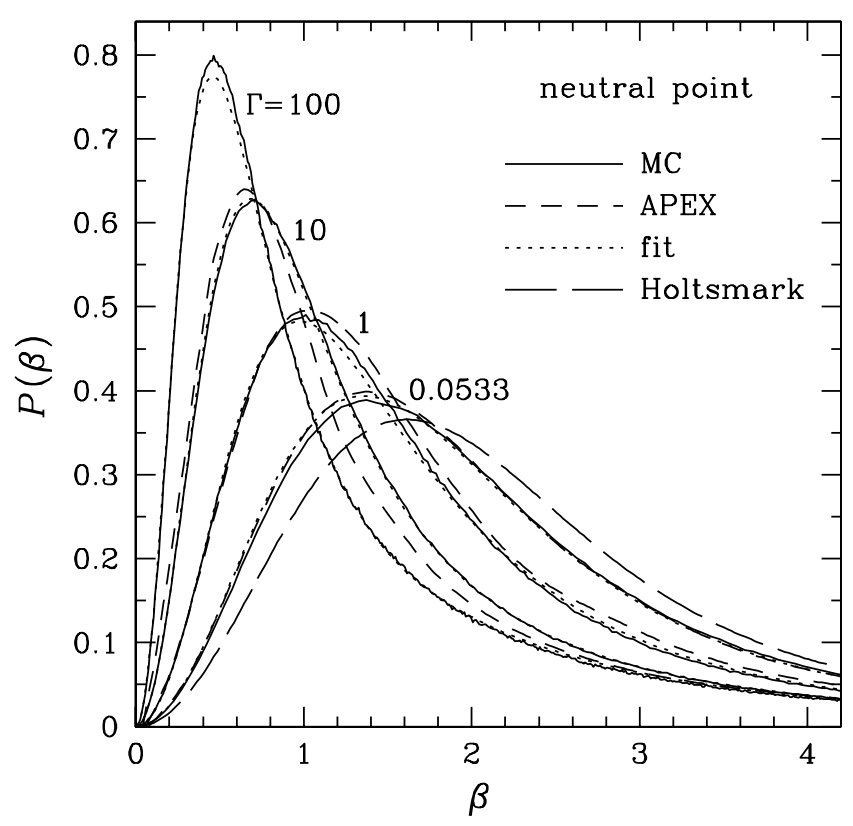

FIG. 3: Microfield distributions produced at a neutral point by ions interacting via the Coulomb potential, for $\Gamma=0.0533$, 1, 10, and 100. Numerical results (MC, solid lines; APEX, short-dashed lines) are compared with analytic approximation (17) (dotted curves). The long-dashed curve reproduces the Holtsmark distribution (11).

For the Holtsmark distribution, $Q_{H}(\beta) \approx 1-0.997 \beta^{-3 / 2}$ at $\beta \rightarrow \infty$. For arbitrary $\beta$, accurate rational-function approximations to $P_{H}(\beta)$ and $Q_{H}(\beta)$ have been constructed by Hummer [35].

With increasing $\Gamma$, the field distribution becomes narrower, as shown in Fig. 迆. The decrease of the most probable value $\beta_{m}$ of the dimensionless field $\beta$, which corresponds to the maximum of $P(\beta)$, can be described by a simple approximate formula

$$
\beta_{m}^{\text {neu }} \approx \frac{1.608+0.24 \sqrt{\Gamma}}{1+0.77 \sqrt{\Gamma}}
$$

The asymptotic behavior of $P(\beta)$ remains power-law, as in the case without Coulomb coupling. This facilitates construction of self-consistent rational approximations to $Q(\beta)$ and $P(\beta)$. We have calculated $P(\beta)$ for various $\Gamma$ from 0 to 10 by the APEX method and for $\Gamma$ up to 100 by the MC technique. Our fit to $Q(\beta)$ reads

$$
Q(\beta)=\frac{q_{0} \beta^{3}-1.33 \beta^{9 / 2}+\beta^{6}}{q_{1}+q_{2} \beta^{2}+q_{3} \beta^{3}-\frac{1}{3} \beta^{9 / 2}+\beta^{6}},
$$

where $q_{n}=\alpha_{n}\left(1+\beta_{n} \sqrt{\Gamma}\right)^{-\gamma_{n}}$, and the parameters $\alpha_{n}$, $\beta_{n}$, and $\gamma_{n}$ are given in Table 1 . $P(\beta)$ is obtained from Eq. (17) by elementary differentiation. At $\Gamma=0$, this differentiation reproduces $P_{H}(\beta)$ at any $\beta$ with a maximum fractional error of $0.24 \%$. At finite $\Gamma$, the difference
TABLE I: Parameters of Eq. (17).

\begin{tabular}{ccccc}
\hline \hline$n$ & 0 & 1 & 2 & 3 \\
\hline$\alpha_{n}$ & 14.600 & 103.20 & 11.127 & 16.178 \\
$\beta_{n}$ & 0.41 & 1.54 & 0.58 & 0.60 \\
$\gamma_{n}$ & 0.707 & 1.64 & 0.572 & 0.915 \\
\hline \hline
\end{tabular}

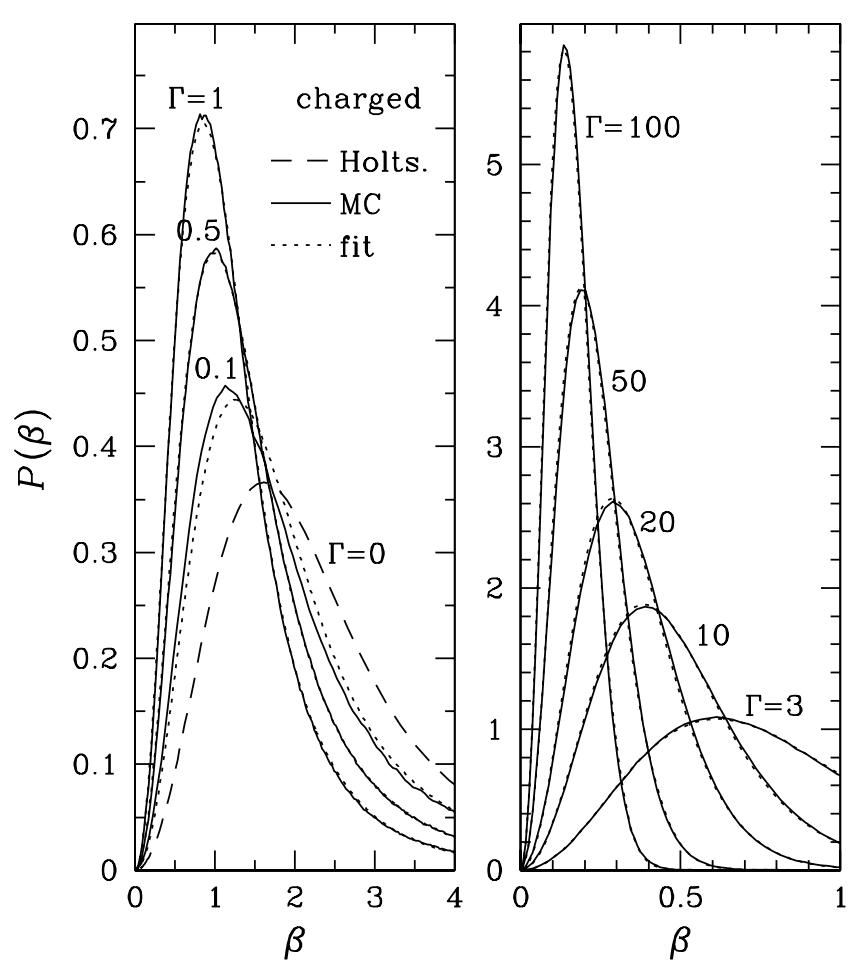

FIG. 4: Microfield distributions produced at a charged test particle by ions interacting via the Coulomb potential at various $\Gamma$ from 0.1 to 100 . MC results (solid lines) are compared with analytic approximation (20) (dotted curves). The Holtsmark distribution $(\Gamma=0)$ is also plotted (dashed line).

between the fit and the MC data increases up to several percent, remaining however not larger than the difference between the MC and APEX results, as one can see in Fig. 3 .

\section{Charged point}

For the charged test particle, the asymptotic behavior of $P(\beta)$ at large $\beta$ is qualitatively different in the cases of zero and non-zero $\Gamma$. When $\Gamma \neq 0$, the power-law decrease of $P(\beta)$ is replaced by an exponential. At moderate $\beta$ and large $\Gamma, P(\beta)$ is approximately described by the Mayer distribution (13). The cumulative function of this distribution is

$$
Q_{M}(\beta, \Gamma)=\operatorname{erf}(\beta \sqrt{\Gamma / 2})-\sqrt{\frac{2 \Gamma}{\pi}} \beta e^{-\Gamma \beta^{2} / 2},
$$



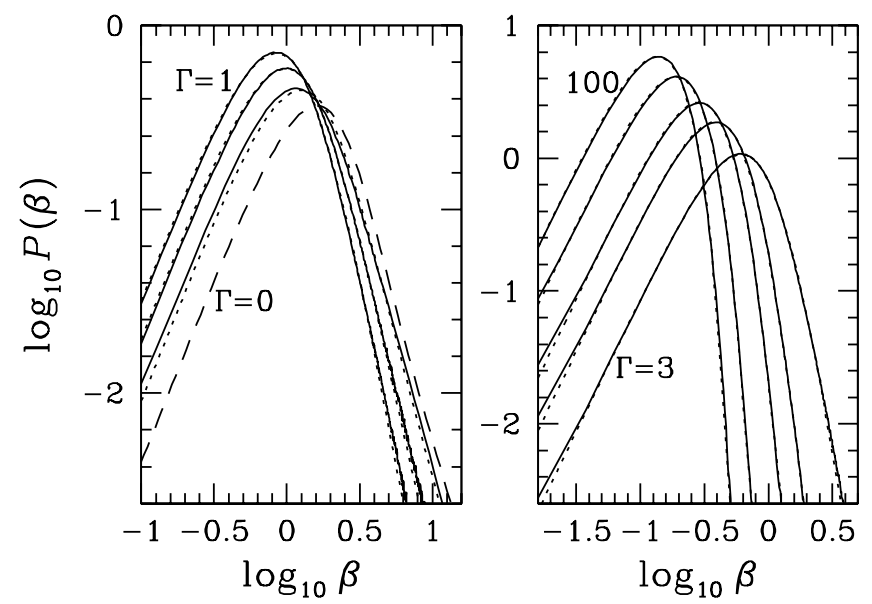

FIG. 5: The same as in Fig. 4 but on the logarithmic scale.

which is easily calculated using, e.g., the highly accurate rational approximation to $e^{x^{2}} \operatorname{erfc}(x)$ in Ref. [36. The most probable value of the microfield provided by this distribution is $\beta_{m}^{M}=\sqrt{2 / \Gamma}$. With increasing $\Gamma, \beta_{m}$ for a charged point decreases faster than $\beta_{m}^{\text {neu }}$ given by Eq. (16). The MC results for $\beta_{m}$ are approximately described by the following modification of $\beta_{m}^{M}$ :

$$
\beta_{m}^{\text {ch }}=\sqrt{2 / \Gamma_{\text {eff }}}, \quad \Gamma_{\text {eff }} \approx 0.774+\Gamma^{1 / 4}+\Gamma .
$$

The microfield probability density at various values of $\Gamma$ is shown in Fig. 1 . The exponential decrease of $P(\beta)$ at large $\Gamma$ is more easily seen in the logarithmic scale (Fig. 5). According to Eq. (14), the exponent at large $\beta$ is proportional to $\sqrt{\beta}$, and not to $\beta^{2}$ as in Eqs. (13) and (18). Nevertheless, it is still possible to construct a self-consistent analytic approximation to $Q(\beta)$ and $P(\beta)$ analogous to Eq. (17). Since the asymptotes are qualitatively different in different coupling regimes, this approximation is more complicated:

$$
Q(\beta)=\frac{Q_{0}(\beta)+0.873 \sqrt{\Gamma} Q_{M}\left(\beta, \Gamma_{\mathrm{eff}}\right)}{1+0.873 \sqrt{\Gamma}},
$$

where

$$
Q_{0}(\beta)=\frac{q \beta^{3} \exp \left(-\Gamma^{\prime} \beta^{1 / 2}\right)+\beta^{6}}{\left[2.25 \pi q\left(1+\Gamma^{0.6}\right)^{-2.75}+15.3 \beta^{2}+1.238 q \beta^{3}+\beta^{9 / 2}\right] \exp \left(-\Gamma^{\prime} \beta^{1 / 2}\right)+\beta^{6}},
$$

$q=9.19+2.178 \Gamma^{1.64}, \quad$ and $\quad \Gamma^{\prime}=\Gamma /\left(1+0.19 \Gamma^{0.627}\right)$.

At $\Gamma=0, \partial Q_{0} / \partial \beta$ reproduces $P_{H}(\beta)$ within $1 \%$. The accuracy deteriorates at $\Gamma \approx 0.1$ but improves again at higher $\Gamma$ : in Figs. 1 and 5 , differences between the fit and MC results are barely visible at $\Gamma=0.5,3,50$, and 100 .

\section{B. Effective screened potential}

In this section, we consider microfield distribution in an electron-screened Coulomb plasma. Assuming that the ions interact via the effective potential (5), we have performed MC simulations of $P(\beta)$ for various values of the screening parameter from $s=0.05$ to $s=3.0$ and the Coulomb coupling parameter from $\Gamma=0.1$ to $\Gamma=100$. In accord with an intuitive expectation, the MC simulations show that the typical fields applied to a test particle are reduced when the electron screening is taken into account. In this case, the probability density $P$ and its cumulative function $Q$ depend on three dimensionless arguments: $\beta, \Gamma$, and $s$. Naturally, analytic approximations in this three-dimensional space become complex and less accurate than at fixed $s=0$; nevertheless we have attempted to construct unified formulas for evaluation of $P(\beta)$ with an accuracy which is sufficient for most applications; the results are presented below.

\section{Neutral point}

The most probable field strength applied to a neutral test particle, evaluated by the MC method, can be parameterized as

$$
\beta_{m}^{\text {neu }}(\Gamma, s) \approx \beta_{m}^{(0)}(s) \frac{1+0.15 \sqrt{\Gamma}}{1+0.77(1+s) e^{-1.5 s} \sqrt{\Gamma}},
$$

where

$$
\beta_{m}^{(0)}(s) \approx\left[0.622+0.25 s e^{s}\right]^{-1} .
$$

Equation (22) extends Eq. (16) to the case where $s \neq$ 0 . It is valid for the whole considered range of plasma parameters, $\Gamma \leq 100$ and $s \leq 3$.

The reduction of the typical microfield strength applied to a charged test particle, when the screening is taken into account, is illustrated in Fig. 6, where the dashed lines correspond to the case of a neutral test particle, which we consider in this section. At a constant $\Gamma, \beta_{m}$ is a monotonically decreasing function of $s$. The dependence of $\beta_{m}$ on $\Gamma$ is less obvious. At small values of $s, \beta_{m}$ 


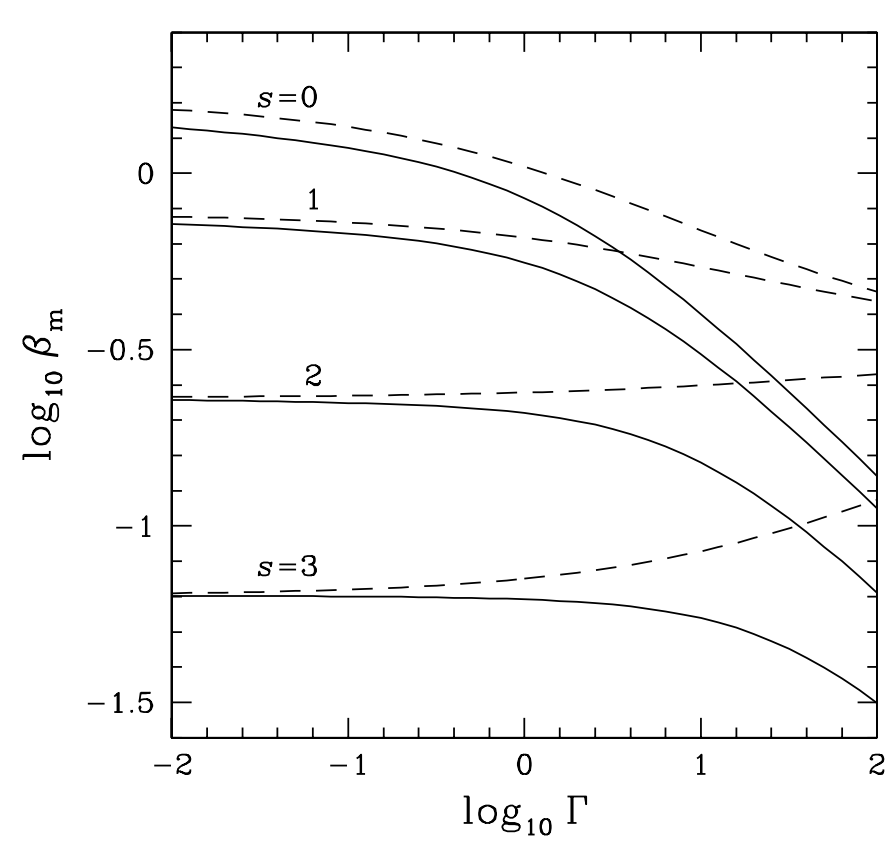

FIG. 6: The "most probable" field strength [normalized according to Eq. (8)] as a function of the Coulomb coupling parameter $\Gamma$ at four values of the screening parameter $s$. Dashed lines: neutral point; solid lines: charged point.

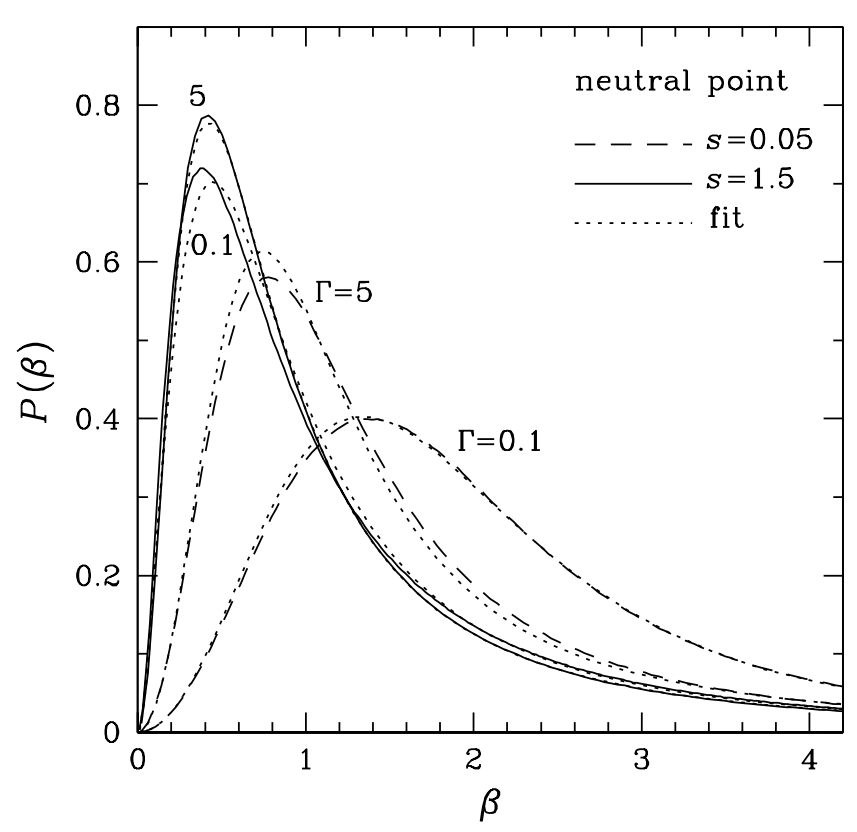

FIG. 7: Microfield distributions produced at a neutral point in a plasma by ions interacting via the screened Coulomb potential for two values of the screening parameter $s$ (dashed lines: $s=0.05$; solid lines: $s=1.5$ ) and two values of the Coulomb coupling parameter $\Gamma$. The analytic approximation (Appendix B) is shown by dotted lines.

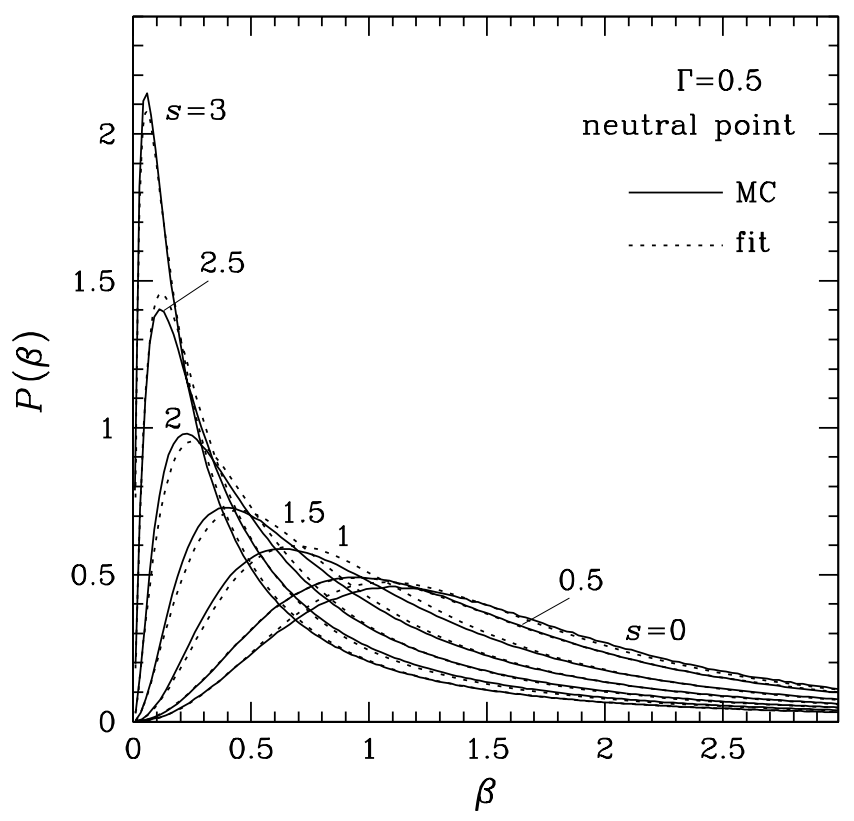

FIG. 8: Microfield distributions produced at a neutral point for the coupling parameter $\Gamma=0.5$, for 7 values of the screening parameter $s$ marked near the curves. MC results (solid lines) are compared with the analytic approximation (dotted lines).

decreases monotonically with increasing $\Gamma$. However, the opposite is observed when $s$ is large: in this case, $\beta_{m}$ increases with growing $\Gamma$. This implies that the most probable field strength depends on $s$ stronger at small $\Gamma$ and weaker at large $\Gamma$.

The modification of the probability density profile with variation of the plasma parameters $\Gamma$ and $s$ is shown in Figs. 17 10. In the most important range of the coupling parameter, $\Gamma \lesssim 10$, the dependence of $P(\beta)$ on $\Gamma$ becomes slow, as the screening becomes sufficiently strong. For example, in Fig. 7 we observe a significant modification of $P(\beta)$ at $s=0.05$, when $\Gamma$ increases from 0.1 to 5 , whereas $P(\beta)$ is only slightly modified with the same increase of $\Gamma$, if $s=1.5$.

On the other hand, the profile of $P(\beta)$ strongly depends on the value of the screening parameter $s$ (Fig. \&), especially if $s \gtrsim 1$. In this case, the most probable field is reduced drastically; simultaneously, the distribution acquires a long "tail," which shows that the values of $\beta \gg \beta_{m}$ occur more often than in the $s=0$ case. Figure 9, which presents the same dependences on the logarithmic scale, clearly reveals the two limiting power laws, $P \propto \beta^{2}$ and $\beta^{-5 / 2}$ at small and large $\beta$ values, respectively, in agreement with Sec. II . We see that these limits are approached considerably more slowly in the case of strong screening.

A comparison of Figs 9 and 10 shows that $P$ is less sensitive to $s$, when $\Gamma$ is large, in accord with the aforementioned property of $\beta_{m}$. Nevertheless, even at $\Gamma \gg 1$, 


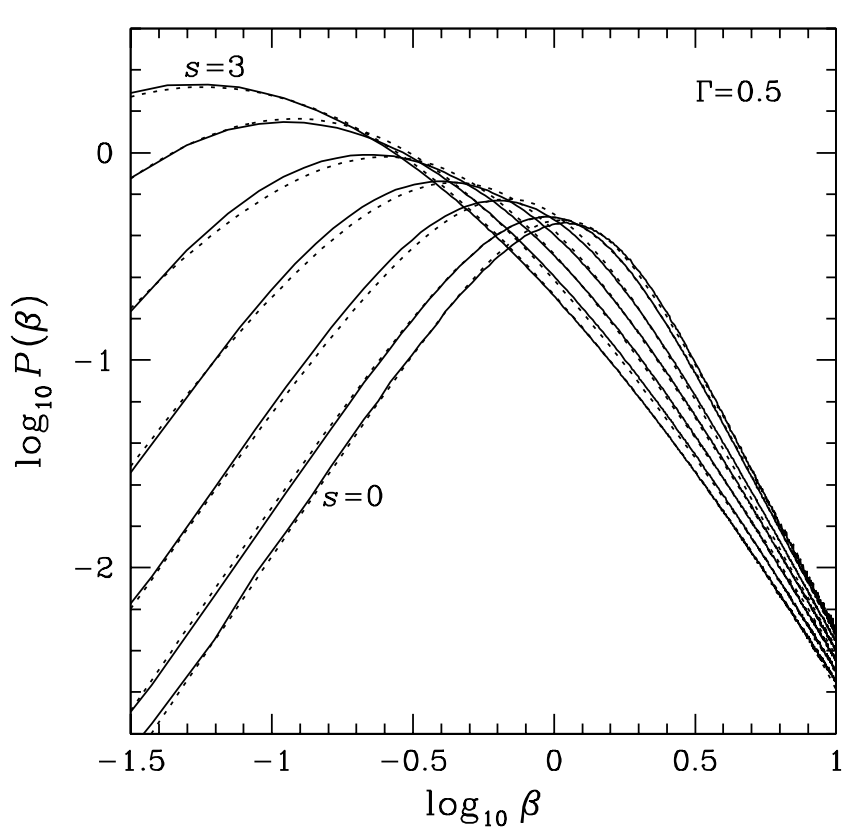

FIG. 9: The same as in Fig. 8 on the logarithmic scale.

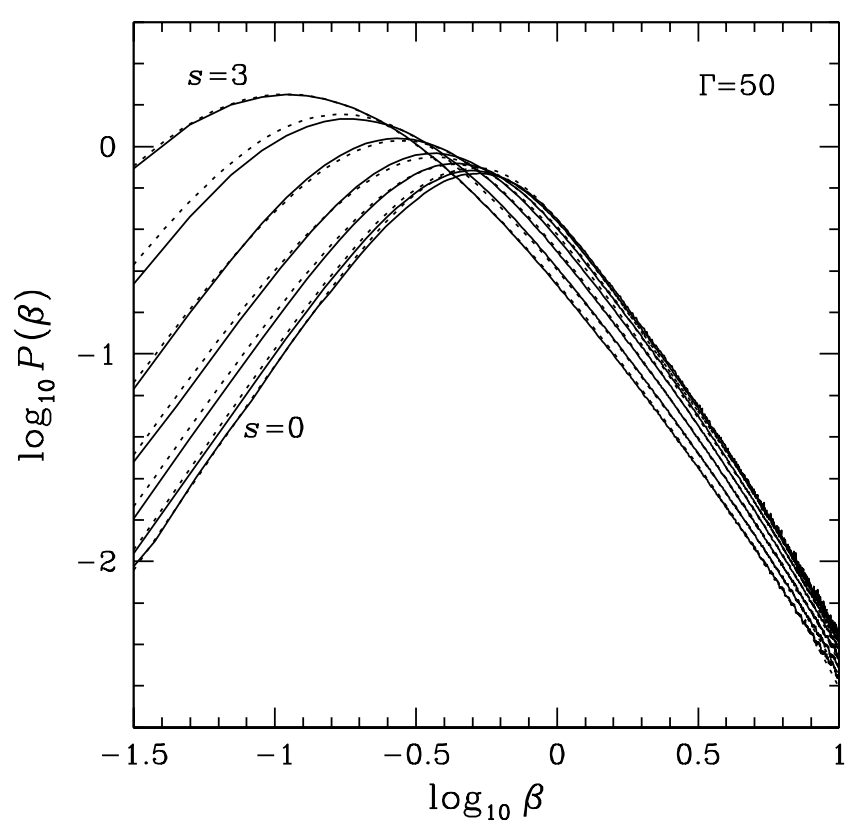

FIG. 10: The same as in Fig. 1 but for $\Gamma=50$.

the sensitivity of $P$ with respect to $s$ remains essential.

It is possible to construct a fitting formula to the probability function $Q(\beta)$ analogous to Eq. (17), taking into account the screening. Such parametric approximation is given in Appendix B and is shown in Figs. $7 \sqrt{10}$ by dotted lines. Although less accurate than the fits presented in Sect. III A, this approximation reproduces well

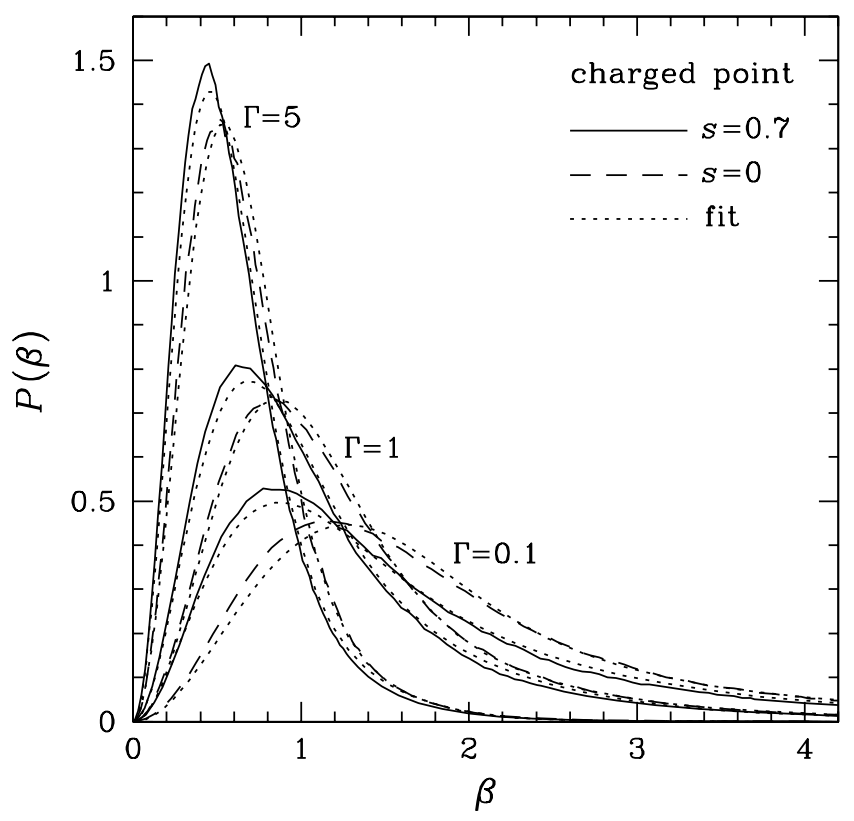

FIG. 11: Microfield distributions produced at a charged test particle by ions interacting via the unscreened (dashed lines) and screened Coulomb potential (solid lines; the screening parameter $s=0.7$ ), for $\Gamma=0.1$, 1 , and 5 . The analytic approximation given in Appendix $\mathrm{d}$ is also shown for all cases (dotted curves).

the numerical results obtained by the MC simulation.

\section{Charged point}

Consider now the microfield distribution created by plasma ions at a point where one of these ions is placed, assuming that the Coulomb interaction is screened according to Eq. (5). As well as in the case of a neutral point, the screening lowers typical microfield values. Dependence of the most probable field $\beta_{m}$ on $s$ and $\Gamma$ can be approximated by a simple expression

$$
\beta_{m}^{\mathrm{ch}}(\Gamma, s)=\beta_{m}^{(0)}(s)\left[1+\frac{\Gamma^{1 / 4}+\Gamma}{0.774+0.54 s e^{s}}\right]^{-1 / 2},
$$

where $\beta_{m}^{(0)}(s)$ is given by Eq. (23). At $s=0$, Eq. (24) reproduces Eq. (19). The dependence of $\beta_{m}$ on $\Gamma$ at various values of $s$ is plotted in Fig. 6 by solid lines. Unlike the case of a neutral point considered in the preceding section, $\beta_{m}^{\text {ch }}$ decreases monotonically with increasing $\Gamma$ at any given value of $s$.

The modification of the probability density $P(\beta)$ with increasing $s$ or $\Gamma$ is illustrated by Figs. 11 14. The dependence on the coupling parameter $\Gamma$ remains qualitatively the same as without screening: with the increase of $\Gamma$, the typical field strengths become lower, and the distribution $P(\beta)$ becomes narrower. The increase of $s$ also 


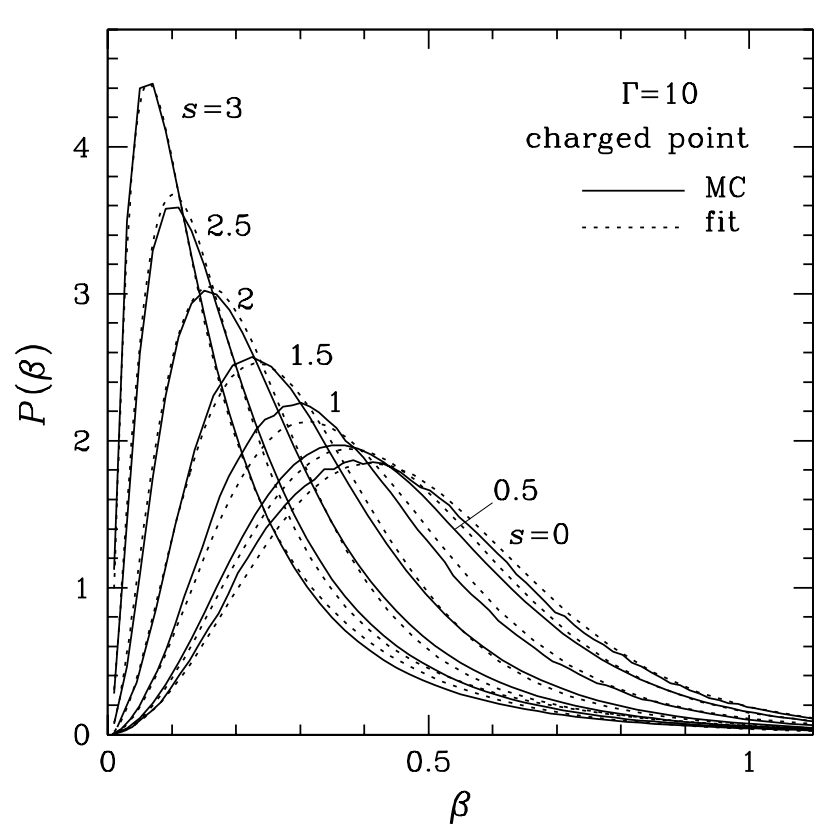

FIG. 12: Microfield distributions produced at a charged test particle for the coupling parameter $\Gamma=10$, for the values of the screening parameter $s$, marked near the curves. MC results (solid lines) are compared with the analytic approximation given in Appendix $\mathrm{O}$ (dotted lines).

shifts the peak of $P$ to smaller $\beta$. At $s>1$, however, the latter shift is accompanied by a striking modification of the shape of the function $P$ : a fast growth at small $\beta$ is followed by a slow, gradual decrease at $\beta>\beta_{m}$.

As in the case of the Coulomb potential, the limiting behavior of $P(\beta)$ at $\beta \gg \beta_{m}$ changes from power law at $\Gamma=0$ to the exponential decrease $\alpha \beta^{-5 / 2} e^{-\Gamma \sqrt{\beta}}$ at $\beta \rightarrow \infty$. This limiting law is reached very slowly, if $s$ is large, as clearly seen in the logarithmic scale (Fig. 13). When $\beta$ is moderately large and $\Gamma \gg 1$, the decrease is approximately Gaussian, $\propto e^{-\Gamma \beta^{2} / 2}$.

The rich variety of the shapes of $P(\beta)$ (depending on $s$ and $\Gamma$ ) complicates significantly the construction of a fitting formula. In this case, a unified fit to $Q$ and $P$ (like those presented in the previous sections) would become too cumbersome. Therefore we have chosen to construct an analytic approximation to the function $P$ only. Whenever necessary, $Q$ can be found by numerical integration [Eq. (15)].

Our approximate formula is presented in Appendix Q. Its quality is revealed by Figs. 11 14, where the fit is compared with results of the MC simulations. The typical accuracy of several percent at $s \lesssim 1.5$ is expected to be sufficient for most applications. At $s>1.5$ the accuracy deteriorates, and at $s>2$ the asymptotic behavior at large $\beta$ is not reproduced, as one can see from the logarithmic plots (Fig. 13). At such strong screening, the fit still may be used for evaluation of $P(\beta)$ not too far

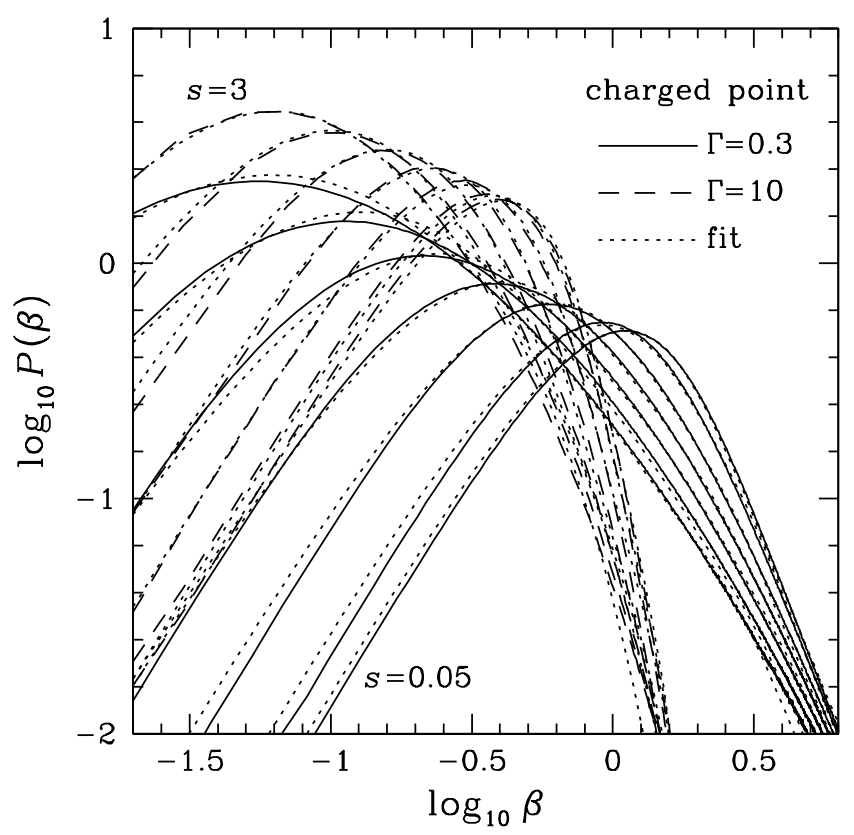

FIG. 13: Comparison of distributions $P(\beta)$ at two values of $\Gamma$ (solid lines: $\Gamma=0.3$; dashed lines: $\Gamma=10$ ) and seven values of $s(s=0.05,0.50,1.04,1.5,2.0,2.5$, and 3.0, from right to left) on the logarithmic scale. Dotted lines show the approximation (Appendix G).

from $\beta_{m}$. Indeed, on the linear scale (Figs. 12, 14) the difference between the fit and the MC results appears to be small even at $s=3$.

Figure 14 allows one to compare the screening effects in the two cases of a neutral and charged test particle. Since $\Gamma$ is greater than unity, the difference between $P(\beta)$ functions in the two cases is large at $s=0.05$ (i.e., for a nearly Coulomb potential), in agreement with Sec. IIIA. With the increase of $s$, however, the difference becomes smaller near the peak of $P(\beta)$. The positions of the peaks for a neutral and charged points almost coincide at $s>$ 1 , and the difference in their heights is caused by the fact that, in the case of a neutral point, $P(\beta)$ decreases much more slowly at large $\beta$, and therefore the region of $\beta \gg \beta_{m}$ gives a larger contribution to the normalization integral, than it does in the case of a charged point.

\section{SUMMARY}

We have calculated microfield distributions at neutral and charged test particles in a one-component plasma of ions, interacting via Coulomb potential, in various regimes from weak to strong coupling. The MC and APEX methods of calculation yield similar distributions, in agreement with previously known results [16. Selfconsistent elementary-function approximations for the field probability density $P(\beta)$ and its cumulative distribution $Q(\beta)$ are constructed in the two cases of a neutral 


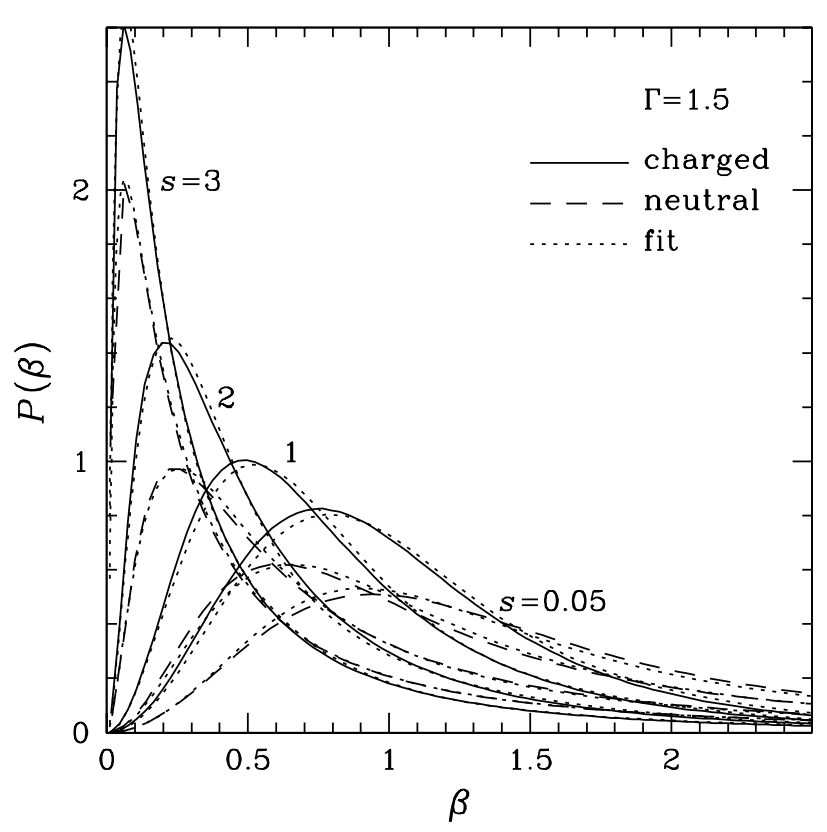

FIG. 14: Comparison of the probability density distributions $P(\beta)$ at a neutral (dashed lines) and charged (solid lines) point at $\Gamma=1.5$ and for four values of $s$. Dotted lines show the approximations in Appendixes B and $\mathrm{C}$.

and charged point, for a Coulomb coupling parameter $\Gamma$ varying from 0 to $10^{2}$.

Furthermore, MC calculations of the microfield distribution have been performed for the screened Coulomb interaction, using the model of ions interacting via the Debye-like (Yukawa) effective potential, with an effective screening length as a second independent parameter. The dimensionless screening parameter $s$ [Eq. (8)] varies from 0 to 3 . The whole set of numerical results for $P(\beta)$ at various values of the coupling and screening parameters is approximated by analytic expressions.

The obtained results can be used in theoretical models of optical spectra and equations of state of Coulomb plasmas.

\section{Acknowledgments}

A. P. thanks V. S. Filinov for useful discussion and the theoretical astrophysics group at the Ecole Normale Supérieure de Lyon for hospitality. The work of A. P. has been partially supported by RFBR Grant No. 99-0218099.

\section{APPENDIX A: HIGH-FIELD ASYMPTOTIC EXPRESSION FOR THE MICROFIELD DISTRIBUTION}

In this Appendix, we outline the derivation of the strong-field asymptotic limit of Eq. (9). For brevity, we adopt the convention that all lengths and radius vectors are measured in units of the ion-sphere radius $a$.

We take advantage of the fact that, for Coulomb interactions, high-field contributions are produced by nearest particles. This well-known result has been investigated in Ref. [13. We assume (i) that the microfield at $\boldsymbol{r}_{0}=\mathbf{0}$ is dominated by the contribution of the nearest neighbor ion located at $\boldsymbol{r}_{1}$, and (ii) that only the potential of this ion contributes to the potential energy. Then Eq. (9) can be approximated by

$$
P(\beta) \sim P_{\mathrm{as}}(\beta)=4 \pi \beta^{2} \frac{\int_{\Omega} d \boldsymbol{r}_{1} \int_{r_{2}>r_{1}} d \boldsymbol{r}_{2} \ldots \int_{r_{N}>r_{1}} d \boldsymbol{r}_{N} e^{-\Gamma v\left(r_{1}\right)} \delta\left(\boldsymbol{\beta}-\nabla v\left(r_{1}\right)\right)}{\int_{\Omega} d \boldsymbol{r}_{1} \int_{r_{2}>r_{1}} d \boldsymbol{r}_{2} \ldots \int_{r_{N}>r_{1}} d \boldsymbol{r}_{N} e^{-\Gamma v\left(r_{1}\right)}} .
$$

Here, $\Omega$ is the total volume of the system, and $v(r)=$ $V(r) a /(Z e)^{2}$ is the reduced potential, so that $\Gamma v(r)=$ $V(r) / k_{B} T$. In our units, $\Omega=\frac{4}{3} \pi N$.

Taking into account that $\int_{\Omega} d \boldsymbol{r}=\Omega, \int_{r_{2}>r_{1}} d \boldsymbol{r}_{2}=$ $\Omega\left(1-r_{1}^{3} / N\right)$, and $\lim _{N \rightarrow \infty}\left(1-r_{1}^{3} / N\right)^{N}=e^{-r_{1}^{3}}$, we obtain

$$
P_{\text {as }}(\beta)=\beta^{2} \frac{\int_{\Omega} e^{-r^{3}-\Gamma v(r)} \delta(\boldsymbol{\beta}-\nabla v(r)) d \boldsymbol{r}}{\int_{0}^{\infty} r^{2} e^{-\Gamma v(r)} d r} .
$$

The coordinate transformation $\boldsymbol{u}=\nabla v(|\boldsymbol{r}|)=$ $\tilde{\beta}(r) \boldsymbol{r} / r$ with the Jacobian $J=\left(r^{2} / \tilde{\beta}^{2}\right)|d r / d \tilde{\beta}|$ yields

$$
P_{\text {as }}(\beta)=\frac{1}{K} r^{2}\left|\frac{d r}{d \tilde{\beta}}\right|_{\tilde{\beta}=\beta} e^{-r^{3}-\Gamma v(r)}
$$

with $\beta=\tilde{\beta}(r)=|d v(r) / d r|$ and $K=\int_{0}^{\infty} r^{2} e^{-r^{3}-\Gamma v(r)} d r$.

For a Coulomb potential, $v(r)=1 / r$ and $\beta=1 / r^{2}$, so that

$$
P_{\text {as }}(\beta) \sim \frac{\beta^{-5 / 2} \exp \left(-\Gamma \beta^{1 / 2}-\beta^{-3 / 2}\right)}{2 \int_{0}^{\infty} \beta^{\prime-5 / 2} \exp \left(-\Gamma{\beta^{\prime}}^{1 / 2}-\beta^{\prime^{-3 / 2}}\right) d \beta^{\prime}} .
$$

For a Yukawa potential $v(r)=e^{-s r} / r$, we have

$$
P(\beta)=\frac{1}{K} \frac{r^{2} \exp \left[-r^{3}-\Gamma e^{-s r} / r\right]}{(2+s r+2 / s r)\left(s / r^{2}\right) e^{-s r}},
$$

where $r$ should be determined from the equation

$$
\beta=(1+s r) \frac{e^{-s r}}{r^{2}},
$$


and

$$
K=\int_{0}^{\infty} r^{2} \exp \left[-r^{3}-\Gamma e^{-s r} / r\right] d r .
$$

Equation (A5) is considerably more complicated than Eq. (A4); it can be compared to Hooper's formulation [13. On the other hand, the simpler Eq. (A4) or its generalization, Eq. (14), can be sufficiently accurate for most applications. This has been verified by comparing with MC results [24, 34] and is illustrated in Fig. 2.

\section{APPENDIX B: APPROXIMATION TO THE PROBABILITY FUNCTION OF MICROFIELDS AT A NEUTRAL POINT}

In this Appendix we present a fitting formula to the probability function $Q(\beta)$, Eq. (15)), for a neutral point in a plasma with Coulomb coupling and screening.

At every pair of $\Gamma$ and $s$ values, we derive a Padé approximation to the microfield probability function,

$$
Q(\beta)=\frac{a_{0} \beta^{3}-2 \beta^{9 / 2}+\beta^{6}}{a_{1}+a_{2} \beta+a_{3} \beta^{2}+a_{4} \beta^{3}-\beta^{9 / 2}+\beta^{6}} .
$$

This expression ensures that, when its derivative is taken, the limits $P(\beta) \propto \beta^{2}$ at $\beta \rightarrow 0$ and $P(\beta) \approx 1.5 \beta^{-5 / 2}$ at $\beta \rightarrow \infty$ are reproduced. At arbitrary $\beta$, an agreement with $\mathrm{MC}$ results is provided by an appropriate choice of the fitting parameters $a_{0}-a_{4}$. The latter parameters, in turn, can be approximated as functions of $\Gamma$ and $s$ :

$$
\begin{aligned}
a_{0}= & \frac{97 s^{2}+1.29 s^{7}}{1+3.1 \times 10^{-3} s^{5}}+\left(59+8.1 s^{2}\right) g \\
& g \equiv \sqrt{0.08+\Gamma}, \\
a_{1}= & \frac{1.16}{1+0.188 s^{6}}\left[1+\frac{103 g^{\alpha(s)}}{1+0.33 s}\right] \\
& \alpha(s) \equiv \frac{0.068+0.038 s^{7}}{1+0.030 s^{7}} \\
a_{2}= & \frac{95 s}{1+6 \times 10^{-3} s^{7}}+1.2 s^{2} g \\
a_{3}= & 27 s^{3}+36 g \\
a_{4}= & \frac{1.894+s}{2+s} a_{0} .
\end{aligned}
$$

This approximation has been checked for the whole range of the plasma parameters for which the MC simulations were performed, i.e., at $0 \leq \Gamma \leq 100$ and $0 \leq s \leq 3$. In the case of purely Coulomb potential $(s=0)$, however, Eq. (17) should be used as more accurate.

\section{APPENDIX C: APPROXIMATION TO THE PROBABILITY DENSITY OF MICROFIELDS AT A PLASMA ION}

In this Appendix we present an analytic approximation to the probability density $P(\beta)$ of electric microfield at an ion in an electron-screened Coulomb plasma.
At every $\Gamma$ and $s$, we write

$$
P(\beta) \approx \frac{\beta^{2}}{S_{N}}\left[A e^{-a \beta^{\alpha}}+B e^{-b \beta^{\gamma}}+\frac{e^{-\Gamma \beta^{1 / 2}}}{1+c \beta^{9 / 2}}\right],
$$

where $S_{N}$ is the normalization constant. For the latter constant, we have

$$
S_{N}=A \frac{\Gamma(3 / \alpha)}{\alpha a^{3 / \alpha}}+B \frac{\Gamma(3 / \gamma)}{\gamma b^{3 / \gamma}}+\Gamma^{-6} F\left(c / \Gamma^{9}\right),
$$

where $\Gamma(3 / \alpha)$ and $\Gamma(3 / \gamma)$ are the Gamma-function values which are easily calculated (e.g., Ref. [36]), and

$$
F(y) \equiv \int_{0}^{\infty} \frac{x^{2} e^{-\sqrt{x}}}{1+y x^{9 / 2}} d x .
$$

For the latter integral, we have constructed an approximation,

$$
\begin{aligned}
& F(y) \approx\left(1+\frac{4 \pi}{9 \sqrt{3}} y^{1 / 9}\right) \\
& \times\left[\frac{1}{240}+0.849 y^{1 / 3}+3.2 y^{5 / 9}+2.43 y^{2 / 3}+y^{7 / 9}\right]^{-1} .
\end{aligned}
$$

Expression ( $\sqrt{\mathrm{C} 4})$ fits the integral $(\overline{\mathrm{C} 3})$ within $0.8 \%$. This is sufficient for evaluation of $Q(\beta)$ and $P(\beta)$ in most applications. The accuracy of Eq. (C4) may be insufficient, however, if the values of $[1-Q(\beta)]$ at $\beta \gg \beta_{m}$ are of interest. In this case, the normalization constant can be evaluated numerically according to Eqs. (C2) and (C3).

Equation (C1) ensures that $P(\beta) \propto \beta^{2}$ at $\beta \rightarrow 0$. Moreover, it also ensures the correct limiting behavior at $\beta \rightarrow \infty$, Eq. (14), with $\tilde{\Gamma}=\Gamma$, provided that $\alpha$ and $\gamma$ are both greater than 0.5 . With the choice of parameters presented below, this is the case at $0 \leq s \lesssim 2.3$ (any $\Gamma$ ), which covers the whole range of values typically encountered in stellar and laboratory dense plasmas.

We have parameterized $A, a, \alpha, B, b$, and $\gamma$ in Eq. (C1) as functions of $\Gamma$, having the same form at any $s$ :

$$
\begin{aligned}
A & =A_{1} \frac{1+A_{4} \sqrt{\Gamma}}{1+A_{2} \Gamma^{2}+A_{3} \Gamma^{4}}, \\
a & =a_{0}+\Gamma / 2 \\
\alpha & =\frac{\alpha_{1}+2 \alpha_{2} \sqrt{\Gamma}}{1+\alpha_{2} \sqrt{\Gamma}} \\
B & =\frac{B_{1}}{1+B_{2} \Gamma^{2}+B_{3} \Gamma^{4}}, \\
b & =b_{0}+\Gamma / 4 \\
\gamma & =\frac{\gamma_{1}+1.5 \gamma_{2} \sqrt{\Gamma}}{1+\gamma_{2} \sqrt{\Gamma}} .
\end{aligned}
$$

Then the parameters of these expressions $\left(A_{n}, B_{n}, a_{0}\right.$, $b_{0}, \alpha_{1}, \alpha_{2}, \gamma_{1}$, and $\gamma_{2}$ ) and the parameter $c$ of Eq. (C1) 
have been approximated by analytic functions of $s$ :

$$
\begin{aligned}
A_{1}= & 0.59+2540 s^{4}+3 s^{14}, \\
A_{2}= & \frac{0.55+10 s^{0.5}+2 s^{4.5}}{1+20 s^{0.5}}, \\
A_{3}= & 2.17 \times 10^{-3} s^{5}, \\
A_{4}= & 14.8 /\left[1+117 s^{3.5}\right], \\
& a_{0}=1.15+2 s^{1.8}, \\
\alpha_{1}= & 0.1+1.1 /\left(1+0.145 s^{3}\right), \\
\alpha_{2}= & \frac{5.4}{1+20 s^{2}}+\frac{1.1}{1+14 s^{0.35}}, \\
B_{1}= & 0.386+300 s^{2}+1.1 s^{9.5}, \\
B_{2}= & 0.038+0.79 s^{0.75}, \\
B_{3}= & \frac{3.7 \times 10^{-3} s^{5.5}}{1+4 \times 10^{-3} s^{9}},
\end{aligned}
$$

$$
b_{0}=\left(1+0.54 s^{2.5}\right) /(1+0.07 s),
$$

$$
\begin{aligned}
& \gamma_{1}=0.1+1.1 /\left(1+0.174 s^{2.5}\right) \\
& \gamma_{2}=\frac{5.4}{1+21 s^{1.5}}+\frac{1.1}{1+19 s^{0.16}}
\end{aligned}
$$

$$
c=\frac{0.097}{1+210 s^{2.5} \exp \left(-1.3 s^{1.5}\right)} .
$$

The high powers of $s$ in some of these equations effectively describe the strong $s$-dependence of the shape of the function $P(\beta)$ at $s \gtrsim 1$.

As noted in Sec. III B 2, this approximation is valid at $\Gamma \leq 100$ and $s \leq 2$, but it can be also used at $2<s \leq 3$, provided that $\bar{\beta}$ is not larger than $\approx 10 \beta_{m}^{\mathrm{ch}}$.
[1] H. R. Griem, Spectral line broadening by plasmas (Academic Press, New York, 1974).

[2] References related to the spectral line broadening are available at http://physics.nist.gov/PhysRefData/Linebr/html/reffrm0.html (J. R. Fuhr and H. R. Felrice) and http://mesunb.obspm.fr/travaux/STRP1E.html (J. R. Fuhr and A. Lesage).

[3] Z. Jiang et al., Phys. Plasmas 2, 1702 (1995); D. Gilles and O. Peyrusse, J. Quant. Spectrosc. Radiat. Transfer 53, 647 (1995).

[4] Y. Vitel, M. El Bezzari, L. G. D'yachkov, and Yu. K. Kurilenkov, Phys. Rev. E 58, 7855 (1998).

[5] D. G. Hummer and D. Mihalas, Astrophys. J. 331, 794 (1988); D. Mihalas, W. Däppen, and D. G. Hummer, ibid. 331, 815 (1988); W. Däppen, D. Mihalas, D. G. Hummer, and B. W. Mihalas, ibid. 332, 261 (1988).

[6] A. Nayfonov, W. Däppen, D. G. Hummer, and D. Mihalas, Astrophys. J. 526, 451 (1999).

[7] A. Y. Potekhin, Phys. Plasmas 3, 4156 (1996).

[8] C. A. Iglesias, H. E. DeWitt, J. L. Lebowitz, D. MacGowan, and W. B. Hubbard, Phys. Rev. A 31, 1698 (1985).

[9] C. Stehlé and S. Jacquemot, Astron. Astrophys. 271, 348 (1993).

[10] J. Holtsmark, Ann. Phys. (Leipzig) 58, 577 (1919).

[11] M. Baranger and B. Mozer, Phys. Rev. 115, 521 (1959); B. Mozer and M. Baranger, ibid. 118, 626 (1960).

[12] C. F. Hooper, Jr., Phys. Rev. 149, 77 (1966).

[13] C. F. Hooper, Jr., Phys. Rev. 169, 193 (1968).

[14] H. Hoffman and O. Theimer, Astrophys. J. 127, 477 (1958).

[15] H. Mayer, Los Alamos Scientific Laboratory Report No. LA-647, 1947 (unpublished).

[16] C. A. Iglesias, J. L. Lebowitz, and D. MacGowan, Phys. Rev. A 28, 1667 (1983).
[17] C. A. Iglesias and J. L. Lebowitz, Phys. Rev. A 30, 2001 (1984).

[18] C. A. Iglesias et al., J. Quant. Spectrosc. Radiat. Transfer 65, 303 (2000).

[19] F. J. Rogers, J. Chem. Phys. 73, 6272 (1980).

[20] G. Chabrier J. Phys. (Paris) 51, 57 (1990).

[21] D. Gilles and A. Angelié, Ann. Phys. (Paris), Coll. 3, 11, 157 (1986).

[22] R. Stamm, B. Talin, E. L. Pollock, and C. A. Iglesias, Phys. Rev. A 34, 4144 (1986).

[23] D. Gilles and C. Stehlé, J. Phys. II (Paris) 5, 75 (1995).

[24] D. Gilles, CEA Report of 04/03/1997 (unpublished).

[25] J.-M. Caillol and D. Gilles, J. Stat. Phys. 100, 905 (2000).

[26] A. V. Demura, D. Gilles, and C. Stehlé, J. Quant. Spectrosc. Radiat. Transfer 54, 123 (1995); C. Stehlé, D. Gilles, and A. V. Demura, Eur. Phys. J. D 12, 355 (2000).

[27] M. S. Murillo, D. P. Kilcrease, and L. A. Collins, Phys. Rev. E 55, 6289 (1997).

[28] Yu. K. Kurilenkov and V. S. Filinov, High Temp. 18, 509 (1980).

[29] H. M. Antia, Astrophys. J. Suppl. Ser. 84, 101 (1993).

[30] P. P. Ewald, Ann. Phys. (Leipzig) 64, 253 (1921); see also C. Kittel, Introduction to Solid State Physics (Wiley, New York, 1986), Appendix B.

[31] S. G. Brush, H. L. Sahlin, and E. Teller, J. Chem. Phys. 45, 2102 (1966).

[32] M. Lewis and H. Margeneau, Phys. Rev. 109, 842 (1958).

[33] D. Paris, CEA Report of 13/8/1969 (unpublished).

[34] I. Gharbi, Ph.D. thesis, Université Paris VI, 1995.

[35] D. G. Hummer, J. Quant. Spectrosc. Radiat. Transfer 36, 1 (1986).

[36] W. H. Press, S. A. Teukolsky, W. T. Vetterling, and B. P. Flannery, Numerical Recipes in Fortran, 2nd ed. (Cambridge U. Press, Cambridge, UK, 1992). 